\section{Androgenentzug per Pflaster?}

\section{Östrogenpflaster zum Androgenentzug scheinen bei Prostatakarzinom- patienten ebenso effektiv zu sein wie eine Therapie mit GnRH-Agonisten. Dies legt zumindest eine Phase-II-Studie aus Großbritannien nahe.}

E ür die Androgendeprivation beim Prostatakarzinom werden gegenwärtig in erster Linie Agonisten oder Blocker des $\mathrm{GnRH}$ („gonadotropin releasing hormone") eingesetzt. Diese Therapie ist allerdings durch langfristige Risiken belastet. Dazu gehören Osteoporose, Dyslipidämien und Typ-2-Diabetes. Britische Forscher haben an 250 Patienten mit lokal begrenztem oder metastasiertem Prostatakarzinom die Effektivität eines Östrogenpflasters (100 mg/24 h) mit der Standardtherapie verglichen.

In der multizentrischen Phase-II-Studie senkten beide Strategien den Testosteronspiegel in etwa gleichem Maße, aber nur, wenn in der Östrogengruppe die Dosis „stimmte“, das heißt, wenn mit vier Pflastern begonnen wurde, die zweimal wöchentlich gewechselt wurden. Nach vier Wochen konnten die Patienten auf drei Pflaster reduzieren, wenn die Testosteronkonzentration auf Kastrationsniveau
(1,7 nmol/l oder weniger) gesunken war. Dieses hatten in der Östrogengruppe nach drei Monaten $92 \%$ der Teilnehmer erreicht, in der GnRH-Gruppe $93 \%$. Die anfängliche Strategie mit drei Östrogenpflastern zu Beginn, die dann auf zwei reduziert wurden, hatte dagegen nur in 69 \% der Fälle zum Erfolg geführt.

Östrogen blockiert die HypothalamusHypophysen-Achse. Bei oraler Verabreichung steigt unter Östrogen das Risiko für Thrombembolien. Diese Gefahr ist bei transkutaner Applikation deutlich geringer. Zu kardiovaskulären Ereignissen, darunter auch Schlaganfälle und venöse Thrombembolien, kam es dennoch bei etwa $10 \%$ der Patienten, die ein Pflaster verschrieben bekommen hatten (in der GnRH-Gruppe waren es $7 \%$ ). Der Unterschied war wegen des weiten Konfidenzintervalls jedoch nicht signifikant. In der Östrogengruppe hatte zudem die Hälfte der Betroffenen das Pflaster seit mindestens 30 Tagen abgesetzt und war zu GnRH-Analoga gewechselt. Im Vorteil waren die Patienten mit Östrogenpflaster bei der Nüchternglukose und dem Gesamtcholesterin. Diese Werte waren in der GnRH-Gruppe um 0,33 beziehungsweise $0,20 \mathrm{mmol} / \mathrm{l}$ gestiegen, in der Pflastergruppe um 0,16 und $0,23 \mathrm{mmol} / \mathrm{l}$ gesunken.

Dagegen führte die Östrogentherapie deutlich häufiger zu einer Gynäkomastie (75\% gegenüber 19\%), und auch leichte Hautprobleme traten häufiger auf (42\% gegenüber $13 \%$ ).

Fazit: Das Östrogenpflaster ist möglicherweise eine kostengünstige Alternative zum Androgenentzug mit GnRH-Analoga. Die Patienten können die Pflaster selbst anlegen, zudem vermeiden sie damit einige Nebenwirkungen, welche die GnRH-Therapie mit sich bringt.

Dr. Elke Oberhofer

Langley RE et al. Cardiovascular outcomes in patients with locally advanced and metastatic prostate cancer treated with luteinisinghormone-releasing-hormone agonists or transdermal oestrogen: the randomised, phase 2 MRC PATCH trial (PR09). Lancet Oncol 2013; 14: 306-16

\section{Prostatakarzinom: Erfolg der Strahlentherapie steht erst nach 15 Jahren fest}

\section{Prostatakrebs lässt sich durch eine Kombination von interner und externer Strahlentherapie dauerhaft gut unter Kontrolle halten. Dennoch kann es selbst nach 15 Jahren noch Rückfälle geben.}

n einer US-amerikanischen Studie waren 3.546 an Prostatakrebs erkrankte Männer (Alter im Median 65 Jahre) in den Jahren 1984 bis 2000 zunächst mit der Implantation von Jod-125-Seeds behandelt worden. Anschließend erhielten die Patienten eine externe Bestrahlung. Die Nachbeobachtungszeit mit halbjährlichen Untersuchungsterminen betrug bis zu 26 Jahre. Das Besondere an der Studie: Statt mit dem üblichen Rezidivkriterium nach Strahlentherapie, PSA-Nadir plus 2, wurde die Rückkehr des Tumors anhand der Maßgabe beurteilt, die nach einer radikalen Prostatektomie gilt: PSA-Anstieg über 0,2 ng/ $\mathrm{ml}$ mit Bestätigung durch einen Kontroll- test. Lag der Nadir von Hause aus über 0,2 ng/ml, bezeichnete der erste Anstieg über diesen Wert den Rückfall.

Unter dieser Maßgabe lag der Anteil der Männer, die krankheitsfrei überlebten, nach zehn, 15, 20 und 25 Jahren bei $75 \%$, $73 \%, 73 \%$ und $73 \%$. Von den Männern, die nach 1995 transperineale Seeds erhalten hatten, überstanden sogar 79\% die folgenden 15 Jahre ohne Rezidiv. Nach Risikoklassen betrachtet, betrug das 15-Jahres-Überleben $92 \%$ (niedriges Risiko), $73 \%$ (mittleres Risiko) und $42 \%$ (hohes Risiko). Der späteste Rückfall ereignete sich 15,5 Jahre nach Therapiebeginn; nach 15 Jahren war bei dem Mann noch ein PSA-Wert unter $0,2 \mathrm{ng} / \mathrm{ml}$ gemessen worden. Spätrezidive, definiert als negativer PSA-Nachweis im zehnten Jahr mit PSA-Anstieg danach, traten bei 31 Patienten auf. Dabei zeigten sich mitunter aggressive Tumoren, entgegen der naheliegenden Annahme, späte Rezidive sprächen für einen eher glimpflichen Verlauf. Drei der Männer mit späten Rückfällen wurden binnen 36 Monaten hormonpflichtig; dabei hatte die PSA-Bestimmung sechs bis zwölf Monate vor dem Rezidiv noch einen Wert von $0,1 \mathrm{ng} / \mathrm{ml}$ oder darunter ergeben.

Fazit: Prostatakarzinomrezidive können auch noch nach 15 Jahren stabil niedrigen PSA-Werten auftreten. Dr. Robert Bublak

Critz FA et al. 25-Year Disease-Free Survival Rate After Irradiation for Prostate Cancer Calculated with the Prostate Specific Antigen Definition of Recurrence Used for Radical Prostatectomy. J Urol 2013; 189: 878-83 